November 4, 2018

\title{
HOMOTOPY AND DUALITY IN NON-ABELIAN LATTICE GAUGE THEORY
}

\author{
ROMAIN ATTAL
}

\begin{abstract}
We propose an approach of lattice gauge theory based on a homotopic interpretation of its degrees of freedom. The basic idea is to dress the plaquettes of the lattice to view them as elementary homotopies between nearby paths. Instead of using a unique $G$-valued field to discretize the connection 1-form, $A$, we use an Aut $(G)$-valued field $U$ on the edges, which plays the role of the 1 -form $\operatorname{ad}_{A}$, and a $G$-valued field $V$ on the plaquettes, which corresponds to the Faraday tensor, $F$. The 1-connection, $U$, and the 2-connection, $V$, are then supposed to have a 2-curvature which vanishes. This constraint determines $V$ as a function of $U$ up to a phase in $Z(G)$, the center of $G$. The 3-curvature around a cube is then Abelian and is interpreted as the magnetic charge contained inside this cube. Promoting the plaquettes to elementary homotopies induces a chiral splitting of their usual Boltzmann weight, $w=v \bar{v}$, defined with the Wilson action. We compute the Fourier transform, $\widehat{v}$, of this chiral Boltzmann weight on $G=S U_{3}$ and we obtain a finite sum of generalized hypergeometric functions. The dual model describes the dynamics of three spin fields : $\lambda_{P} \in \widehat{G}$ and $m_{P} \in \widehat{Z(G)} \simeq \mathbb{Z}_{3}$, on each oriented plaquette $P$, and $\varepsilon_{a b} \in \widehat{\operatorname{Out}(G)} \simeq \mathbb{Z}_{2}$, on each oriented edge $(a b)$. Finally, we sketch a geometric interpretation of this spin system in a fibered category modeled on the category of representations of $G$.
\end{abstract}

\section{CONTENTS}

1. Introduction 1

2. Homotopies on a lattice 2

2.1. From plaquettes to homotopy generators 2

2.2. Iterated paths 4

3. Gauge fields and curvatures 5

4. Partition function and duality 7

4.1. Duality transformation 8

4.2. The dual connection 10

5. Conclusions and perspectives 11

Appendix A. $S U_{3}$ and its characters 11

Appendix B. Free dynamics on the weight lattice and Fourier's inversion formula 13

Appendix C. The Fourier transform of the chiral Boltzmann weight on $S_{3}$

References

\section{INTRODUCTION}

A discretization of Yang-Mills gauge theory on a lattice has been defined by $\mathrm{K}$. Wilson in order to find an analytic criterion for the confinement of quarks [17]. This approach suggests that confinement is a consequence of the non-Abelian nature of the structure group (usually $G=S U_{N}$ ). The gauge invariant quantities, i.e. the physical observables of the theory, are obtained by averaging products of traces of holonomies of a connection on a vector bundle, along spin networks. The latter are directed graphs whose edges carry a representation of $G$ and whose vertices carry a homomorphism from the tensor product of the incoming representations to the tensor product of the outgoing ones. These observables can be expanded as power series whose coefficients are sums over surfaces made of plaquettes $[17,6,11]$. In this framework, the correspondence between weak and strong coupling is expressed by a Fourier transformation on the space of gauge fields and is a non-Abelian generalization of the Kramers-Wannier duality [9]. In [13], the dual theory has been formulated as a spin-foam model on the dual lattice. In the present work, we propose to make a further step in the study of non-Abelian duality by using homotopic methods.

Our paper is organized as follows. In Section 2, we define the dressed cells of our lattice in order to view them as elementary homotopies. They can be seen as the generators of the spaces of iterated paths. In Section 3, we define the gauge fields of our system and their partition function, in which the 2-curvature is forced to vanish. Since Wilson's action is proportional to the real part of the trace (in the fundamental representation of $G$ ) of the holonomy along a little square loop, the Boltzmann weight, $w$, of an unoriented plaquette is the product of two complex conjugate weights, $v$ and $\bar{v}$, each 
one being associated to an orientation of this plaquette. This chiral splitting allows to associate independant representations to the 2-cells of the dual lattice which correspond to oppositely oriented plaquettes. In Section 4, we express the partition function of the dual model in terms of three fields of representations (or spin fields) taking their values in $\widehat{G}, \widehat{Z(G)}$ and $\widehat{\operatorname{Out}(G)}$. Finally, in Section 5, we draw some conclusions and future prospects.

In the appendices, we collect some mathematical results. In App. A, we recall some useful results about the structure of $S U_{3}$ and its characters. In App. B, we give a dual interpretation of Wilson's action by proving that $\widehat{w}$ satisfies a diffusion equation on the weight lattice of $S U_{3}$. In a Minkowskian space-time, this corresponds to the motion of a free particle following a discretized Schrödinger's equation. The application of the spectral theorem to the corresponding discrete Laplacian provides us with a direct proof of Fourier's inversion formula. In App. C, we compute explicitely the Fourier coefficients of the chiral Boltzmann weight $v$ : this is a linear combination of generalized hypergeometric series.

\section{Homotopies ON A LATTICE}

2.1. From plaquettes to homotopy generators. The building blocks of a gauge theory on a lattice are the cells of this lattice. At the beginning, we consider them as mere subsets of the space in which the lattice is embedded. So, let $\mathbb{Z}^{4}$ be embedded in the four dimensional euclidian space $\mathbb{R}^{4}$, endowed with its canonical basis $\left(e_{i}\right)_{1 \leq i \leq 4}$. We will use the following notations :

$$
\begin{aligned}
A & =\{1,2,3,4\} \\
\widetilde{A} & =A \cup\{0\} \cup(-A) \\
A_{p} & =\left\{i=\left(i_{1}, \cdots, i_{p}\right) \in A^{p}: i_{1}<\cdots<i_{p}\right\} \\
e_{-i} & =-e_{i} \quad \forall i \in \widetilde{A} \\
u & =\left(e_{1}+e_{2}+e_{3}+e_{4}\right) / 2
\end{aligned}
$$

Definition : For each $p \in\{0,1,2,3,4\}, C_{p}$ denotes the set of bare (unoriented) $p$-cells

$$
C_{p}:=\mathbb{Z}^{4} \times A_{p}
$$

If we choose a characteristic map $\left(f_{\sigma}: B^{p} \rightarrow \mathbb{R}^{4}\right)$ to parametrize homeomorphically each $p$-cell $\sigma$ by the unit ball $B^{p} \subset \mathbb{R}^{p}$, then we obtain a cell complex $C=\left(C_{p}\right)_{1 \leq p \leq 4}$. The geometric realization of $\sigma \in C_{p}$ is the image of $f_{\sigma}$, noted $|\sigma|$, and the $p$-skeleton of $C$ is the sub-complex $C^{p}=\left(C_{q}\right)_{q \leq p}$. The terms "vertex", "link", "plaquette", "cube" and "hypercube" will denote the sets $|\sigma| \subset \mathbb{R}^{4}$, for $\sigma \in C_{0}, C_{1}, C_{2}, C_{3}$ and $C_{4}$, respectively. In order to take into account the two possible orientations of each cell, we adjoin a sign and we define the sets of oriented cells by

$$
C^{\prime}{ }_{p}:=C_{p} \times\{-1,+1\}
$$

Definition : The dual of $C^{\prime}$ is the complex $C^{* *}=\left(C_{p}^{\prime *}\right)_{0 \leq p \leq 4}$ defined by

$$
C_{p}^{\prime *}:=\left(\mathbb{Z}^{4}+u\right) \times A_{p} \times\{-1,+1\}
$$

We have a duality map

$$
\begin{aligned}
*: C^{\prime} & \longrightarrow C^{\prime *} \\
\sigma=(a, i) & \longmapsto \sigma^{*}=\left(a+u-e_{i^{*}}, i^{*}\right)
\end{aligned}
$$

where $i^{*}=\left(i_{1}^{*}, \cdots, i_{4-p}^{*}\right)$ is the ordered multi-index defined by 


$$
\left\{i_{1}^{*}, \cdots, i_{4-p}^{*}\right\}=A \backslash\left\{i_{1}, \cdots, i_{p}\right\} \quad \text { and } \quad i_{1}^{*}<\cdots<i_{4-p}^{*}
$$

and $e_{i^{*}}=\left(e_{i_{1}^{*}}+\cdots+e_{i_{p}^{*}}\right) / 2$.

When we work with a non-Abelian structure group, we need to dress the bare cells with a supplementary structure in order to interpret them as elementary homotopies.

Definition : The set $\widetilde{C}_{p}$ of homotopic p-cells is defined by

$$
\widetilde{C}_{p}:=C^{\prime}{ }_{0} \times \widetilde{A}^{p}
$$

Therefore, we have the inclusions

$$
C_{p} \subset C^{\prime}{ }_{p} \subset \widetilde{C}_{p}
$$

A homotopic 0 -cell is simply a vertex with a sign. A homotopic 1 -cell $\ell=(a, i, \varepsilon) \in \widetilde{C}_{1}$ has a 0 -source and a 0 -target defined by

$$
\begin{aligned}
& s_{0}(a, i, 1)=t_{0}(a, i,-1)=a \\
& t_{0}(a, i, 1)=s_{0}(a, i,-1)=a+e_{i}
\end{aligned}
$$

The fluxes associated to the various ways of sweeping a given plaquette are related by a representation of the symmetry group of the square. Indeed, the 2 -cells which start at $\ell$ are of the form $(\ell, j, 1)$ with $j \in \widetilde{A}$ and the 2-cells which contain $\ell$ are obtained by the action of the following group :

$$
\begin{aligned}
E & :=D_{4} \times D_{1} \\
D_{4} & =\left\langle r, s \mid r^{4}=s^{2}=(r s)^{2}=1\right\rangle \\
D_{1} & =\left\langle\iota \mid \iota^{2}=1\right\rangle
\end{aligned}
$$

$D_{4}$ acts on $\{a, b, c, d\}$ by

$$
\begin{aligned}
s(a) & =d & & s(b)=c \\
s(c) & =b & & s(d)=a \\
r(a) & =d & & r(b)=a \\
r(c) & =b & & r(d)=c
\end{aligned}
$$

and this induces an action of $E$ on $\widetilde{C}_{2}$ :

$$
\begin{aligned}
r(x, i, j, \varepsilon) & =\left(x+e_{i}, j,-i, \varepsilon\right) \\
s(x, i, j, \varepsilon) & =\left(x+e_{i},-i, j, \varepsilon\right) \\
\iota(x, i, j, \varepsilon) & =(x, i, j,-\varepsilon)
\end{aligned}
$$

Therefore, the subset of non-degenerate homotopic 2-cells is a $D_{4}$-bundle over $C_{2}$.

The conjugation map on $\widetilde{C}_{2}$ can also be extended to $\widetilde{C}$ :

$$
\begin{aligned}
\widetilde{C}_{p} & \longrightarrow \widetilde{C}_{p} \\
\tau=(x, j, \varepsilon) & \longmapsto \bar{\tau}=(x, j,-\varepsilon)
\end{aligned}
$$


2.2. Iterated paths. The loop approach of lattice gauge theories $[17,15,8]$ suggests to work in the spaces of iterated paths of our lattice. We propose the definition given below, but a more careful treatment should use the notion of cellular homotopy $n$-groupoid.

Definition : The set of paths is defined by

$$
\mathcal{P}_{1}:=\left\{\left(a_{0}, \cdots, a_{p}\right): a_{i} \in \mathbb{Z}^{4} \quad \text { and } \quad\left\|a_{i+1}-a_{i}\right\|=1 \quad \text { for } \quad i=0, \cdots, p-1\right\}
$$

Let $\gamma=\left(a_{0}, \cdots, a_{p}\right)$ and $\gamma^{\prime}=\left(a_{0}^{\prime}, \cdots, a_{q}^{\prime}\right)$ be two paths. If $t_{0}(\gamma)=s_{0}\left(\gamma^{\prime}\right)$, i.e. $a_{p}=a_{0}^{\prime}, \gamma$ and $\gamma^{\prime}$ can be composed by concatenation :

$$
\gamma^{\prime} \cdot \gamma=\left(a_{0}, \cdots, a_{p}, a_{1}^{\prime}, \cdots, a_{q}^{\prime}\right)
$$

The composition of $\gamma$ with the reversed family, $\left(a_{p}, \cdots, a_{0}\right)$, provides a path which is homotopic to $\left(a_{0}, a_{0}\right)$ in $\left|C^{1}\right|$. Similarly, the 0 -source and 0 -target of a 2 -cell $P=\left(a, i_{1}, i_{2}, \varepsilon\right) \in \widetilde{C}_{2}$ are the vertices defined by

$$
\begin{aligned}
& s_{0}(P)=a \\
& t_{0}(P)=a+e_{i_{1}}
\end{aligned}
$$

and its 1-source and 1-target are the paths defined by

$$
\begin{aligned}
& s_{1}\left(a, i_{1}, i_{2},+1\right)=s_{1}\left(a, i_{1}, i_{2},-1\right)=\left(a, a+e_{i_{1}}\right) \\
& t_{1}\left(a, i_{1}, i_{2},+1\right)=s_{1}\left(a, i_{1}, i_{2},-1\right)=\left(a, a+e_{i_{2}}, a+e_{i_{2}}+e_{i_{1}}, a+e_{i_{1}}\right)
\end{aligned}
$$

We will write generically

$$
(a, b, c, d)=\left(a, a+e_{j}, a+e_{j}+e_{i}, a+e_{i}\right)
$$

the four corners of $P=(a, i, j, \varepsilon)$ :
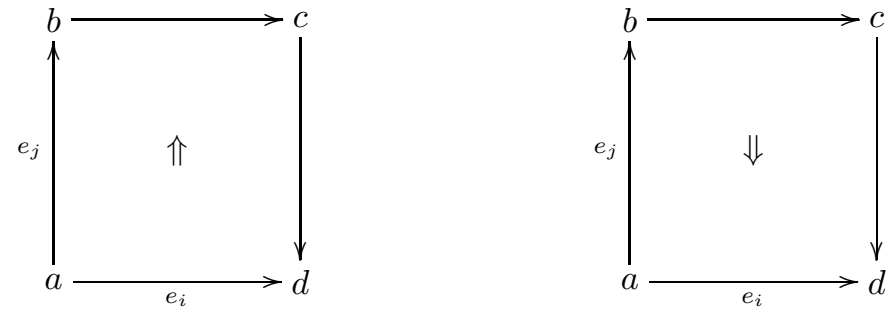

Roughly speaking, if $\varepsilon=+1, P$ represents a $(1 \rightarrow 3)$ homotopy and if $\varepsilon=-1, P$ represents a $(3 \rightarrow 1)$ homotopy. We could use also $(0 \rightarrow 4)$ or $(2 \rightarrow 2)$ homotopies, i.e. diagonal motions, but the set $\widetilde{C}_{2}$ defined above is sufficient to connect all the paths and respects the symmetry of the lattice by moving the paths only along the lattice vectors $\left(e_{i}\right)_{i \in B}$. We allow degenerate 2-cells, with $i_{2}= \pm i_{1}$, which represent tangential motions of the links. Their boundary is homotopic, in $\left|C^{1}\right|$, to a constant path. Therefore, they carry no flux and do not contribute to the dynamics.

With higher dimensional cells, we can also define the sets $\mathcal{P}_{n}$ of $n$-paths as follows.

Definition : For $n>1$, a n-path is a finite family $\left(X_{0}, \cdots, X_{p}\right)$ of $(n-1)$-paths in which the composition of any two successive members is homotopic, in $\left|C^{n-1}\right|$, to the boundary of a $(n-1)$-cell :

$$
\mathcal{P}_{n}:=\left\{\left(X_{0}, \cdots, X_{p}\right): X_{i} \in \mathcal{P}_{n-1} \quad \text { and } \quad \exists \sigma \in C_{n} \quad \text { s.t. } \quad X_{i+1} \circ X_{i}^{-1} \sim_{n-1} \partial \sigma\right\}
$$


If two $k$-paths, say $\Gamma, \Gamma^{\prime} \in \mathcal{P}_{k}$, are homotopic in the $k$-skeleton of $C$, i.e. if they can be joined by a sequence of motions along degenerate $(k+1)$-cells, we will write $\Gamma \sim_{k} \Gamma^{\prime}$.

Definition : For $q<p$, we define the $q$-source and the $q$-target of a $p$-path in the obvious way, so that we have maps

$$
\begin{aligned}
& s_{q p}: \mathcal{P}_{p} \longrightarrow \mathcal{P}_{q} \\
& t_{q p}: \mathcal{P}_{p} \longrightarrow \mathcal{P}_{q}
\end{aligned}
$$

which satisfy

$$
\begin{aligned}
& s_{r q} \circ s_{q p}=s_{r q} \circ t_{q p} \\
& t_{r q} \circ s_{q p}=t_{r q} \circ t_{q p}
\end{aligned}
$$

\section{Gauge Fields And CURvatures}

In the standard approach, the connection is represented (locally) by a map $\left(g: C_{0} \times \widetilde{A} \rightarrow G\right)$ from the set of 1-cells to the structure group, satisfying $g_{\bar{\ell}}=g_{\ell}^{-1}$. Then, to each oriented plaquette with a base point, is associated the holonomy of $g$ along its boundary. However, in the homotopic context, we need to separate the degrees of freedom associated to the 1 -cells from those associated to the 2-cells. This leads us to use two fields

$$
\begin{aligned}
& U: \widetilde{C}_{1} \longmapsto \operatorname{Aut}(G) \\
& V: \widetilde{C}_{2} \longmapsto G
\end{aligned}
$$

which will be called, respectively, the 1-connection and the 2-connection. We will also write

$$
\begin{aligned}
V_{a b c d} & =V_{(a, i, j, 1)} \\
V_{\overline{a b c d}} & =V_{(a, i, j,-1)}
\end{aligned}
$$

These fields are supposed to satisfy the following conditions :

$$
\begin{array}{rll}
U_{b a}=U_{a b}^{-1} & \text { and } & V_{\overline{a b c d}}=V_{a b c d}^{-1} \\
V_{d a b c}=U_{d a}\left(V_{a b c d}\right) & \text { and } & V_{d c b a}=U_{d a}\left(\overline{V_{a b c d}}\right)
\end{array}
$$

which ensure that we have a natural representation of the space of paths. Indeed, the first two conditions mean that the motion along $b a$ (resp. $\overline{a b c d}$ ) is represented by the inverse of the group element corresponding to $a b$ (resp. $a b c d$ ). The third relation means that a rotation of angle $\pi / 2$ applied to $a b c d$ replaces the flux $V_{a b c d} \in G$ by an equivalent flux $V_{d a b c}$ obtained by applying the automorphism $U_{d a}$, which corresponds to the motion of the 0-target along the 1-source of $a b c d$. The second relation in (2) means that the flux swept by the flipped 2-cell $d c b a=s(a b c d)$ is the complex conjugate of the flux swept by $a b c d$, pulled back to $s_{0}(s(a b c d))=t_{0}(a b c d)=d$. Thus, we have only one independent variable $V$ per plaquette, the others being obtained by the action of $U$ or by inversion.

With the 1-connection and the 2-connection, we can define two kinds of curvature: the 2-curvature, defined on the 2-cells, and the 3-curvature, defined on the cubes.

Definition : The 2-curvature $\Phi$ is the map defined by

$$
\begin{aligned}
\Phi: \widetilde{C}_{2} & \longrightarrow \operatorname{Aut}(G) \\
a b c d & \longmapsto \Phi_{a b c d}=I_{V_{a b c d}} U_{a b} U_{b c} U_{c d} U_{d a} \\
\overline{a b c d} & \longmapsto \Phi \overline{a b c d}=\Phi_{a b c d}^{-1}
\end{aligned}
$$


where $I$ denotes the inner automorphism map

$$
\begin{aligned}
I: G & \longrightarrow \operatorname{Aut}(G) \\
g & \longmapsto\left(I_{g}: h \mapsto g h g^{-1}\right)
\end{aligned}
$$

When the lattice spacing goes to zero, $\Phi$ corresponds to the "fake curvature" 2 -form $\nu$ as defined in [5]. Since $r^{4}(a b c d)=$ $a b c d$ and since we want to represent the homotopy along abcd as a flux which depends only on the direction of sweeping, we impose the relation

$$
V_{a b c d}=V_{r^{4}(a b c d)}=U_{a b} U_{b c} U_{c d} U_{d a}\left(V_{a b c d}\right)
$$

which implies the vanishing of the 2-curvature :

$$
\Phi_{a b c d}:=I_{V_{a b c d}} U_{a b} U_{b c} U_{c d} U_{d a}=1_{\text {Aut }(G)} \quad \forall a b c d \in \widetilde{C}_{2}
$$

In order to describe the Yang-Mills-Wilson theory, we must impose another relation between $U$ and $V$, which is a discrete version of Bianchi's identity. Let $Q=\left(a, i_{1}, i_{2}, i_{3}, \varepsilon\right) \in \widetilde{C}_{3}$ be a generic 3-cell :

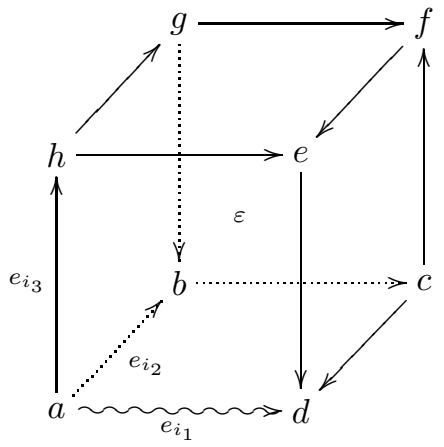

Starting from the path $(a d)=s_{1}(Q)$, if we want to reach the path $(a b c d)=t_{1}(Q)$, we can sweep either the 2-cell $(a d, a b c d)=s_{2}(Q)$ or the five remaining plaquettes, which form $t_{2}(Q)$ with the following sweeping scheme :

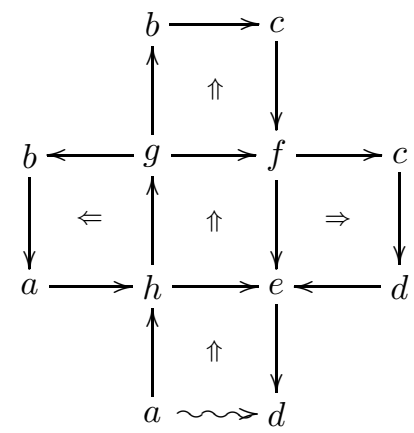

Now we want to represent in $G$ the homotopy leading from $s_{2}(Q)$ to $t_{2}(Q)$. For this purpose, we make the product of the six fluxes (pulled back to $s_{0}(Q)=a$ ) which correspond to the six plaquettes swept in the order defined above and, if $\varepsilon=+1$, we put 


$$
\Omega_{Q}:=U_{a d}\left(V_{\text {defc }}\right) V_{a h e d} U_{a h}\left(V_{h g f e}\right) U_{a h g}\left(V_{g b c f}\right) V_{a b g h} V_{a d c b}
$$

Definition : The 3-curvature is the map

$$
\begin{aligned}
\Omega: \widetilde{C}_{3} & \longrightarrow G \\
Q & \longmapsto \Omega_{Q} \\
\bar{Q} & \longmapsto \Omega_{\bar{Q}}=\Omega_{Q}^{-1}
\end{aligned}
$$

Remark : Bianchi's identity can be stated as the vanishing condition for the 3-curvature $\left(\Omega_{Q}=1_{G}\right)$. However, we don't impose this condition in our present model, because $\Omega_{Q}$ is the magnetic charge inside $Q$ and these topological defects could play a crucial role in the mechanism of quark confinement. In fact, Bianchi's identity is almost satisfied thanks to the following elementary lemma.

Lemma : If the 2-curvature vanishes then the 3-curvature is abelian:

$$
\left(\Phi_{P}=1_{\mathrm{Aut}(G)} \quad \forall P \in \widetilde{C}_{2}\right) \quad \Longrightarrow \quad\left(\Omega_{Q} \in Z(G) \quad \forall Q \in \widetilde{C}_{3}\right)
$$

Let $Q \in \widetilde{C}_{3}$. After the definition of $\Omega_{Q}$, we have

$$
\begin{aligned}
I_{\Omega_{Q}} & =I_{U_{a d}\left(V_{d e f c}\right)} I_{V_{a h e d}} I_{U_{a h}\left(V_{h g f e}\right)} I_{U_{a h g}\left(V_{g b c f}\right)} I_{V_{a b g h}} I_{V_{a d c b}} \\
& =\left(U_{a d} I_{V_{d e f c}} U_{d a}\right) I_{V_{a h e d}}\left(U_{a h} I_{V_{h g f e}} U_{h a}\right)\left(U_{a h g} I_{V_{g b c f}} U_{g h a}\right) I_{V_{a b g h}} I_{V_{a d c b}}
\end{aligned}
$$

Using the relation (3), we obtain

$$
\begin{aligned}
I_{\Omega_{Q}}= & \left(U_{a d} U_{d c} U_{c f} U_{f e} U_{e d} U_{d a}\right)\left(U_{a d} U_{d e} U_{e h} U_{h a}\right)\left(U_{a h} U_{h e} U_{e f} U_{f g} U_{g h} U_{h a}\right) \\
& \left(U_{a h g} U_{g f} U_{f c} U_{c b} U_{b g} U_{g h a}\right)\left(U_{a h} U_{h g} U_{g b} U_{b a}\right)\left(U_{a b} U_{b c} U_{c d} U_{d a}\right) \\
= & 1_{\text {Aut }(G)}
\end{aligned}
$$

Therefore, $\Omega_{Q}$ is in the kernel of $I$, i.e. in the center of $G$.

\section{PARTition FUnCTION AND DUALity}

We are now ready to write down the partition function of this lattice gauge theory defined in terms of homotopies. Let tr denote the trace in the fundamental representation of $G$. Let $\alpha=\left(3 g^{2} T\right)^{-1}$, where $g$ is the Yang-Mills coupling and $T$ denotes the temperature of the four dimensional system. The usual Boltzmann weight

$$
w(M)=\exp \left(2 \alpha \operatorname{Re}\left(\operatorname{tr}\left(M-1_{G}\right)\right)\right)
$$

is associated to an unoriented plaquette. However, since the elementary homotopies along 2-cells carry an orientation, we will use as Boltzmann weight the function $(v: G \rightarrow \mathbb{C})$ defined by

$$
v(M):=\exp \left(\alpha \operatorname{tr}\left(M-1_{G}\right)\right)
$$

We thus have $w=|v|^{2}$. Let $N \in \mathbb{N}$ and let $\Lambda_{N}$ be the box defined by 


$$
\Lambda_{N}:=\left\{x \in \mathbb{R}^{4}:\left|x_{i}\right| \leq N \quad \forall i \in A\right\}
$$

The degrees of freedom contained in this box are described by the maps $U$ and $V$ which satisfy the relations (1) to (3) and which are fixed to unity outside of $\Lambda_{N}$. These maps form compact groups on which we have the product Haar measures, denoted $D U$ and $D V$. The partition function of this system is then defined by

$$
\mathcal{Z}:=\int D U \int D V \prod_{P \in C_{2}^{\prime}} \delta\left(\Phi_{P}\right) v\left(V_{P}\right)
$$

where $\delta\left(\Phi_{P}\right)$ denotes the Dirac distribution at $1_{\mathrm{Aut}(G)}$. Note that the relation $w=v \bar{v}$ allows us to split the Boltzmann weight as

$$
\prod_{P \in C_{2}} w\left(V_{P}\right)=\prod_{P \in C_{2}^{\prime}} v\left(V_{P}\right)
$$

4.1. Duality transformation. Let's apply Fourier's inversion formula

$$
v(M)=\sum_{\lambda \in \widehat{G}} d_{\lambda} \widehat{v}_{\lambda} \bar{\chi}_{\lambda}(M)
$$

to express $\mathcal{Z}$ as the partition function of a spin system :

$$
\begin{aligned}
\mathcal{Z} & =\int D U \int D V\left(\prod_{P \in C_{2}^{\prime}} \delta\left(\Phi_{P}\right) \sum_{\lambda \in \widehat{G}} d_{\lambda} \widehat{v}_{\lambda} \bar{\chi}_{\lambda}\left(V_{P}\right)\right) \\
& =\sum_{\lambda}\left(\prod_{P \in C_{2}^{\prime}} d_{\lambda_{P}} \widehat{v}_{\lambda_{P}}\right)\left(\int D U \int D V \prod_{P \in C_{2}^{\prime}} \delta\left(\Phi_{P}\right) \bar{\chi}_{\lambda_{P}}\left(V_{P}\right)\right)
\end{aligned}
$$

$\widehat{v}$ is computed in App. C. $\lambda$ now denotes a map from the set of oriented 2-cells to $\widehat{G}$ :

$$
\begin{aligned}
\lambda: C_{2}^{\prime} & \longrightarrow \widehat{G} \\
P & \longmapsto \lambda_{P}
\end{aligned}
$$

so that $\lambda_{a b c d}$ and $\lambda_{d c b a}$ are independent degrees of freedom. The constraint of vanishing 2-curvature can be solved as follows. In App. D, we show how Aut $(G)$ can be described as a semi-direct product

$$
\operatorname{Aut}(G) \simeq(G / Z) \rtimes \operatorname{Out}(G)
$$

This isomorphism is canonical and tells us that, for each edge $a b, U_{a b}$ defines a unique outer automorphism $c^{\varepsilon_{a b}}$, where c $\in \operatorname{Out}(G)$ denotes the complex conjugation and $\varepsilon_{a b} \in \mathbb{Z}_{2}=\{0,1\}$. Moreover, we can choose $T_{a b} \in G$ such that

$$
U_{a b}=\left(Z T_{a b}, c^{\varepsilon_{a b}}\right)
$$

where $Z g$ is the class of $g \in G$ in the coset $G / Z$. In terms of $T$ and $\varepsilon$, the 2-curvature becomes

$$
\Phi_{a b c d}=\left(Z V_{a b c d} T_{a b} T_{b c} T_{c d} T_{d a}, c^{\mathrm{d} \varepsilon_{a b c d}}\right)
$$

Since $\Phi_{a b c d}=1_{\mathrm{Aut}(G)}, \varepsilon$ is closed 


$$
\mathrm{d} \varepsilon=0
$$

and, for each choice of $T_{a b}$, there exists an antisymmetric map $\left(m: C_{2}^{\prime} \rightarrow \widehat{Z}\right)$ such that

$$
V_{a b c d}=\exp \left(\frac{2 i \pi}{3} m_{a b c d}\right) T_{a d} T_{d c} T_{c b} T_{b a}
$$

Henceforth, we will suppose that $\varepsilon=0$, i.e. that $U_{a b}$ lies in the connected component of $1_{\mathrm{Aut}(G)}$, because we want $U$ to approximate a continuous field. The phase of $T_{a b}$ is arbitrary and can be shifted freely by a gauge transformation on $m$ which, however, leaves $\Omega$ unchanged :

$$
\begin{aligned}
n: C_{1}^{\prime} & \longrightarrow \mathbb{Z}_{3} \\
(a b) & \longmapsto n_{a b}=-n_{b a} \\
T^{\prime}{ }_{a b} & =e^{\frac{2 i \pi}{3} m_{a b}} T_{a b} \\
m^{\prime}{ }_{a b c d} & =m_{a b c d}+(\mathrm{d} n)_{a b c d} \\
\Omega^{\prime} & =e^{\frac{2 i \pi}{3} \mathrm{~d}^{2} n} \Omega=\Omega
\end{aligned}
$$

We can now express the partition function as a sum over $(\lambda, m)$ constrained by an integral over $T$ :

$$
\mathcal{Z}=\sum_{\lambda m} \int D T \prod_{P \in C_{2}^{\prime}} d_{\lambda_{P}} \widehat{v}_{\lambda_{P}} \bar{\chi}_{\lambda_{P}}\left(V_{P}(T, m)\right)
$$

Then we use the 3-ality $\tau_{\lambda} \in \widehat{Z} \simeq \mathbb{Z}_{3}$, defined by the restriction of $R_{\lambda}$ to $Z=Z(G)$, or by

$$
\tau_{\lambda}=p-q \quad \bmod 3
$$

to separate the variables $m$ and $T$ in the decomposition of $\bar{\chi}_{\lambda_{P}}\left(V_{P}\right)$ :

$$
\bar{\chi}_{\lambda_{P}}\left(V_{P}\right)=e^{\frac{2 i \pi}{3} \tau_{\lambda_{P}} m_{P}} \sum_{q_{a} q_{b} q_{c} q_{d}=1}^{d_{\lambda_{P}}}\left[R_{\lambda_{P}}\left(T_{a b}\right)\right]_{q_{a} q_{b}}\left[R_{\lambda_{P}}\left(T_{b c}\right)\right]_{q_{b} q_{c}}\left[R_{\lambda_{P}}\left(T_{c d}\right)\right]_{q_{c} q_{d}}\left[R_{\lambda_{P}}\left(T_{d a}\right)\right]_{q_{d} q_{a}}
$$

Let $\ell=(a, i) \in C_{1}$ and let $d=a+e_{i}$. Following [13], we evaluate $\int d T_{\ell}$ by collecting all the factors which contain $T_{\ell}$ in the integrand of $\mathcal{Z}$. Let

$$
J_{\lambda \ell} \simeq \prod_{P \supset \ell}\left\{1, \cdots, d_{\lambda_{P}}\right\}
$$

index an orthonormal basis adapted to a decomposition into irreducible components of the tensor product of the representations $R_{\lambda_{P}}$ associated to the twelve oriented 2-cells $P \in C_{2}^{\prime}$ which contain $\ell$ :

$$
\bigotimes_{P \supset \ell} R_{\lambda_{P}}=\bigoplus S_{\lambda_{i}}
$$

Next, we insert the precedent decomposition into the partition function :

$$
\begin{aligned}
\mathcal{Z} & =\sum_{\lambda m}\left(\prod_{P \in C_{2}^{\prime}} d_{\lambda_{P}} \widehat{v}_{\lambda_{P}} e^{\frac{2 i \pi}{3} \tau_{\lambda_{P}} m_{P}}\right)\left(\prod_{\ell \in C_{1}} K_{\lambda \ell}\right) \\
K_{\lambda \ell} & =\sum_{q r \in J_{\lambda \ell}} \int d T_{\ell}\left[\bigotimes_{P \supset \ell} R_{\lambda_{P}}\left(T_{\ell}\right)\right]_{q r}
\end{aligned}
$$


Let $\mathcal{A}=C_{\text {alg }}(G)$ be the algebra generated by the matrix entries of all finite dimensional representations of $G$. We can view a representation $(R: G \rightarrow G L(\mathcal{V}))$ as a vector in the $\mathcal{A}$-module

$$
\mathcal{M}=\operatorname{End}(\mathcal{V}) \otimes_{\mathbb{C}} \mathcal{A}
$$

The integral of $R$ over $G$ provides the orthogonal projector $\mathcal{P}$ onto the $G$-invariant subspace $\mathcal{V}_{0} \subset \mathcal{V}$ :

$$
\int_{G} R=\mathcal{P} \in \operatorname{End}(\mathcal{V}) \subset \mathcal{M}
$$

If we apply this result to $\int d T_{\ell}\left[\otimes R_{\lambda_{P}}\right]$, we obtain

$$
\begin{aligned}
\mathcal{V}_{\lambda \ell} & =\bigotimes_{P \supset \ell} V_{\lambda_{P}} \\
\mathcal{P}_{\lambda \ell} & \in \operatorname{End}\left(\mathcal{V}_{\lambda \ell}\right) \\
K_{\lambda \ell} & =\sum_{q r \in J_{\lambda \ell}}\left[\mathcal{P}_{\lambda \ell}\right]_{q r}
\end{aligned}
$$

Therefore, the partition function of our initial gauge fields is also that of a dual spin system, constrained by the projectors $\mathcal{P}_{\lambda \ell}$ :

$$
\mathcal{Z}=\sum_{\lambda m}\left(\prod_{P \in C_{2}^{\prime}} d_{\lambda_{P}} \widehat{v}_{\lambda_{P}} e^{\frac{2 i \pi}{3} \tau_{\lambda_{P}} m_{P}}\right)\left(\prod_{\ell \in C_{1}} \sum_{q r \in J_{\lambda \ell}}\left[\mathcal{P}_{\lambda \ell}\right]_{q r}\right)
$$

We can define this field theory on the dual lattice, $C^{\prime *}$, by associating each representation $R_{\lambda_{P}}$ to the dual oriented 2-cell $P^{*} \in C^{\prime *}$. The dual of the 2-cells which contain $\ell$ are the 2-cells of $C^{\prime *}$ which form the boundary of the 3-cell $\ell^{*}$ :

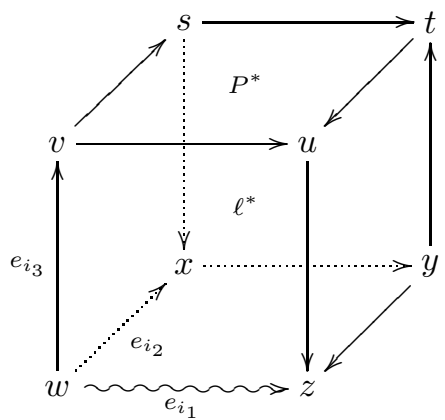

In order to extend the map $\left(P^{*} \mapsto R_{\lambda_{P^{*}}}\right)$ to the set of all 2-cells of the dual lattice, we must represent the action of $D_{4}$ on $C^{\prime *}$ and for this purpose, we need a dual 1-connection. This dual 1-connection must be a family of maps associated to the dual edge-paths and such that the two maps associated, respectively, to the source and target of a dual 2-cell, $P^{*}$, can be related via the conjugation by $R_{\lambda_{P^{*}}}$. This relation is a dual vanishing curvature condition.

4.2. The dual connection. We sketch here very briefly a geometric interpretation of the dual spin system. In the initial gauge theory, we have replaced the usual $G$-valued 1-cochain $\left(g_{a b}\right)$ by the $(G \rightarrow \operatorname{Aut}(G))$-valued 2-cocycle $\left(V_{P}, U_{a b}\right)$. After a duality transformation, the theory is described by a field of representations $\left(R_{\lambda_{P}}\right)$ defined on the set of oriented plaquettes of the dual lattice. The integration over the group variables then constrains the dual 3-curvature to vanish. Let $\mathbf{A}$ denote the category of representations of $G$ over finite dimensional complex vector spaces. The $\mathbf{A}$-valued 2 -form $R$ is closed, i.e. for each cube $Q$ of the dual lattice, there exist non-zero intertwiners between $\bigotimes_{P \subset Q} R_{\lambda_{P}}$ and the trivial representation $\left(R_{0}: G \rightarrow \mathrm{GL}(\mathbb{C})\right):$ 


$$
\bigotimes_{P \subset Q} R_{\lambda_{P}} \leftrightharpoons R_{0}
$$

If Poincaré's Lemma extends to the case of $\mathbf{A}$-valued $p$-forms, then there exists an $\mathbf{A}$-valued 1-form $\rho$ and a field of non-zero intertwiners between $d \rho$ and $R$ :

$$
\rho_{w x} \otimes \rho_{x y} \otimes \rho_{y z} \otimes \rho_{z w} \leftrightharpoons R_{\lambda_{[w x y z]}}
$$

If we integrate over such spin fields, $\rho$, then the dual Bianchi constraint is automatically satisfied. The field $\rho$ can be viewed as a functorial connection in a fibered category with typical fiber $\phi_{x}=\mathbf{A}$, at each site $x$ of the dual lattice. $\rho_{x y}$ then acts by multiplication, say on the left :

$$
\begin{aligned}
\phi_{y} & \longrightarrow \phi_{x} \\
r & \longmapsto \rho_{x y} \otimes r
\end{aligned}
$$

The field $R$ then appears as a flat 2-connection on a 2-stack and $\rho$ is the 1-connection of a stack whose differential is the precedent 2-stack. The construction of these spaces requires a fully categorical approach to lattice gauge theory [12] as well as the extension of the geometry of $G$-gerbes [5] to stacks modeled on tensor categories.

\section{Conclusions And Perspectives}

In the present work, we have laid the basis of a homotopic approach to non-Abelian lattice gauge theories where the field strength represents the homotopies along the 2-cells and the gauge field transports the field strength along the edge-paths. A difference with the usual approach $[17,6,11,8]$ is in the usage of complex weights, $v$ and $\bar{v}$, associated to oppositely oriented 2-cells. We obtain a new closed formula for the Boltzmann weight of the dual spin system which couples three fields of representations : one for $G$, one for its center and one for its group of automorphisms. We have chosen to keep the latter vanishing in order to approximate continuous fields. In this dual spin model appears a projector $\mathcal{P}_{\lambda Q}$ on each 3-cell, $Q$, of the dual lattice. A careful definition shows that this projector depends on the way $\partial Q$ is swept. When $\mathcal{P}_{\lambda Q} \neq 0$, any tensor product of the six representations associated to the six oriented plaquettes of $\partial Q$ admits a non-zero intertwiner onto the trivial representation of $G$. This is the condition of vanishing 2-curvature for the dual spin model, and it is satisfied when, for each plaquette $P$, there exists a non-vanishing morphism of representations between $R_{\lambda_{P}}$ and the curvature $d \rho_{P}$ of a $\operatorname{Rep}(G)$-valued 1-form $\left(\rho_{x y}\right)$. The problem is then to count, for each field of highest weights $\left(\lambda_{P}\right)$, the number of 1-forms $\left(\rho_{x y}\right)$ for which such an intertwiner exists. A suitable version of Poincaré's lemma would imply that this number is non-zero when the 2-form $\left(R_{\lambda_{P}}\right)$ is closed and this number would give the weight of $\rho$ in the expression of the unconstrained partition function. This is the analogue of the volume of the gauge group in the initial theory, formulated in terms of connection and curvature.

The main lesson of the present work is that the spaces adapted to the formulation of non-Abelian duality are fibered categories modeled on categories of $G$-torsors and $G$-modules. We must therefore reformulate the present homotopic approach in a purely categorical language and we hope that these methods will provide a notion of duality adapted to the treatment of strongly coupled gauge theories.

Acknowledgements : I thank Olivier Babelon, Marc Bellon, Robert Oeckl and Hendryk Pfeiffer for useful discussions at various stages of this work.

Appendix A. $S U_{3}$ AND ITS CHARACTERS

We recall here some standard results of group theory [1] to compute the Fourier transform of $v$. Let $T$ be the maximal torus of $\mathrm{SU}_{3}$ whose elements are the diagonal matrices 


$$
D=\left(\begin{array}{ccc}
e^{i \theta_{1}} & 0 & 0 \\
0 & e^{i\left(\theta_{2}-\theta_{1}\right)} & 0 \\
0 & 0 & e^{-i \theta_{2}}
\end{array}\right) \quad \forall \theta_{1}, \theta_{2} \in[0,2 \pi[
$$

Let $\mathfrak{t}$ be the Lie algebra of this torus. Let $L_{1}, L_{2}, L_{3} \in \mathfrak{t}^{*}$ be the linear forms on $\mathfrak{t}$ defined by

$$
L_{i}(D)=D_{i i}
$$

The root system of $t$ is the subset of $\mathfrak{t}^{*}$ defined by

$$
\Phi:=\left\{L_{i}-L_{j}: i, j \in\{1,2,3\}\right\}
$$

The adjoint representation of $\mathfrak{t}$ on $\mathfrak{g}$ provides the Cartan decomposition of $\mathfrak{g}$ into eigenspaces of $\mathfrak{t}$ :

$$
\mathfrak{g}=\mathfrak{t} \oplus\left(\bigoplus_{\alpha \in \Phi} \mathfrak{g}_{\alpha}\right)
$$

$\mathfrak{t}$ corresponds to the 0 eigenvalue and each $\mathfrak{g}_{\alpha}$ is a one-dimensional representation of $\mathfrak{t}$ :

$$
[H, X]=\alpha(H) X \quad \forall H \in \mathfrak{t}, \forall X \in \mathfrak{g}_{\alpha}
$$

We can choose in $\Phi$ a subset $\Pi$ which is a basis of $t$ and such that every root $\alpha \in \Phi$ is a linear combination of elements of $\Pi$ with coefficients which are either all positive or all negative. This is our system of fundamental roots. In the present case, we take

$$
\begin{aligned}
\Pi & =\left\{\alpha_{1}, \alpha_{2}\right\} \\
\alpha_{1} & =L_{1}-L_{2} \\
\alpha_{2} & =L_{2}-L_{3}
\end{aligned}
$$

$\mathfrak{t}$ being endowed with the scalar product induced by the restriction of the Killing form

$$
\left\langle H, H^{\prime}\right\rangle=\operatorname{tr}\left(H H^{\prime}\right) \quad \forall H, H^{\prime} \in \mathfrak{t}
$$

extended to $t^{*}$, we can associate to $\Pi$ the fundamental weights $\omega_{i}$ defined by

$$
\left\langle\omega_{i}, \alpha_{j}\right\rangle=\delta_{i j}
$$

and whose expression in terms of fundamental roots is

$$
\omega_{1}=\frac{2 \alpha_{1}+\alpha_{2}}{3} \quad \text { and } \quad \omega_{2}=\frac{2 \alpha_{2}+\alpha_{1}}{3}
$$

The weight lattice of $S U_{3}$ is generated by $\omega_{1}$ and $\omega_{2}$ :

$$
\Lambda_{w}=\left\{\lambda=p \omega_{1}+q \omega_{2}: p, q \in \mathbb{Z}\right\} \subset \mathfrak{t}^{*}
$$


Each finite dimensional, $\mathbb{C}$-linear, irreducible representation $(R: G \rightarrow G L(V))$ is characterized by the existence of a weight $\lambda \in \Lambda_{w}$, with positive coordinates on $\omega_{1}$ and $\omega_{2}$, and of a vector $u \in V$ such that $R(H)(u)=\lambda(H) u$ for all $H \in \mathfrak{t}$. The set $\widehat{G}$ of isomorphy classes of such representations is then parametrized by two positive integers :

$$
\widehat{G} \simeq\left\{\lambda=p \omega_{1}+q \omega_{2}: p, q \in \mathbb{N}\right\}
$$

\section{Appendix B. Free dynamics on the Weight lattice and Fourier's inversion formula}

We give here a short proof of the fact that the Fourier transform of $w=v \bar{v}$ follows a free dynamics on the weight lattice of $S_{3}$. The definition of $v$ and $w$ implies

$$
\begin{aligned}
\partial_{\alpha} v & =\left(\chi_{10}-3\right) v \\
\partial_{\alpha} w & =\left(\chi_{10}+\chi_{01}-6\right) w
\end{aligned}
$$

Moreover, a straightforward computation shows that

$$
\begin{aligned}
& \chi_{10} \chi_{p q}=\chi_{p+1, q}+\chi_{p, q-1}+\chi_{p-1, q+1} \\
& \chi_{01} \chi_{p q}=\chi_{p, q+1}+\chi_{p-1, q}+\chi_{p+1, q-1}
\end{aligned}
$$

Consequently, $\widehat{v}, \widehat{\bar{v}}$ and $\widehat{w}$ satisfy the following difference/differential equations :

$$
\begin{aligned}
\partial_{\alpha} \widehat{v}_{p q} & =\widehat{v}_{p+1, q}+\widehat{v}_{p, q-1}+\widehat{v}_{p-1, q+1}-3 \widehat{v}_{p q} \\
\partial_{\alpha} \widehat{\bar{v}}_{p q} & =\widehat{\bar{v}}_{p, q+1}+\widehat{\bar{v}}_{p-1, q}+\widehat{\bar{v}}_{p+1, q-1}-3 \widehat{\bar{v}}_{p q} \\
\partial_{\alpha} \widehat{w}_{p q} & =\widehat{w}_{p+1, q}+\widehat{w}_{p, q-1}+\widehat{w}_{p-1, q+1}+\widehat{w}_{p, q+1}+\widehat{w}_{p-1, q}+\widehat{w}_{p+1, q-1}-6 \widehat{w}_{p q}
\end{aligned}
$$

Let $\mathcal{E}$ be the Banach space $\ell^{\infty}\left(\mathbb{N}^{2}, \mathbb{C}\right)$ of bounded families of complex numbers indexed by $\mathbb{N}^{2}$. Let $\mathcal{H}$ be the Hilbert space $\ell^{2}\left(\mathbb{N}^{2}, \mathbb{C}\right)$ of square integrable families of complex numbers indexed by $\mathbb{N}^{2}$ :

$$
\mathcal{E}=\ell^{\infty}\left(\mathbb{N}^{2}, \mathbb{C}\right) \text { and } \mathcal{H}=\ell^{2}\left(\mathbb{N}^{2}, \mathbb{C}\right)
$$

$\mathbb{N}^{2}$ being endowed with the metric induced by the Killing form of $t^{*}$ via the relation (7), we define the corresponding discrete Laplace-Beltrami operator, $\Delta \in B(\mathcal{E})$, by

$$
(\Delta \phi)_{p q}:=\frac{1}{6}\left(\phi_{p+1, q}+\phi_{p, q-1}+\phi_{p-1, q+1}+\phi_{p, q+1}+\phi_{p-1, q}+\phi_{p+1, q-1}\right)-\phi_{p q}
$$

$\Delta$ computes the mean value of a function at the six nearest neighbours of each site $\lambda$ and substracts the value at $\lambda$. In a fundamental dual Weyl chamber, we represent $\Delta$ by the following diagram : 


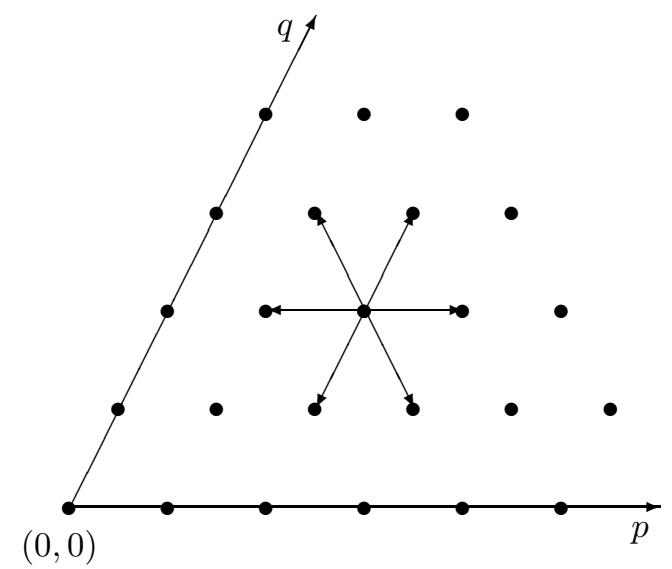

Thus, $\widehat{w}$ satisfies a diffusion equation on $\mathbb{N}^{2} \times \mathbb{R}_{+}$:

$$
\partial_{\alpha} \widehat{w}=6 \Delta \widehat{w}
$$

with the boundary conditions

$$
\widehat{w}_{0 q}=\widehat{w}_{p 0}=0 \quad \text { and } \quad \widehat{w}_{p q}(0)=\delta_{0 p} \delta_{0 q}
$$

The eigenspaces of $\Delta$ in $\mathcal{E}$ are spanned by the eigenfunctions $\left(\phi_{x y}\right)_{x, y \in \mathbb{T}}$ defined by

$$
\begin{aligned}
\phi_{x y}: \mathbb{N}^{2} & \longrightarrow \mathbb{C} \\
(p, q) & \longmapsto \phi_{x y}(p, q)=x^{p} y^{q}
\end{aligned}
$$

and the corresponding eigenvalues, $\left(\mu_{x y}\right)_{x, y \in \mathbb{T}}$, are

$$
\mu_{x y}=\frac{1}{6}\left(x+x^{-1}+y+y^{-1}+x / y+y / x\right)-1
$$

The spectrum, $\sigma(\Delta)$, of our Laplacian is the closure of $\mu\left(\mathbb{T}^{2}\right)$ in $\mathbb{R}$ :

$$
\sigma(\Delta)=\overline{\mu\left(\mathbb{T}^{2}\right)}=\left[-\frac{4}{3}, 0\right]
$$

Since $\Delta$ is a bounded self-adjoint operator on $\mathcal{H}$, we can apply the spectral theorem [19] and expand $\widehat{v}_{p q}$ on the eigenfunctions $\phi_{x y}$ of $\Delta$

$$
\begin{aligned}
\widehat{v}_{p q} & =\int_{\mathbb{T}^{2}} d x d y\left\langle\phi_{x y}, \widehat{v}\right\rangle \phi_{x y}(p, q) \\
\left\langle\phi_{x y}, \widehat{v}\right\rangle & =\sum_{p, q \geq 1} \overline{\phi_{x y}(p, q)} \widehat{v}_{p q} \\
\phi_{x y}(p, q) & =d_{p q} \chi_{p q}(M)
\end{aligned}
$$

where $M=\operatorname{diag}\left(x, y,(x y)^{-1}\right) \in T$, to obtain Fourier's inversion formula for the class functions : 


$$
v=\sum_{\lambda \in \widehat{G}} \widehat{v}_{\lambda} d_{\lambda} \bar{\chi}_{\lambda}
$$

\section{Appendix C. The Fourier transform of the chiral Boltzmann weight on $S U_{3}$}

The characters of the irreducible, finite dimensional representations of $G$ can be computed by applying Weyl's character formula as follows. For all $p, q \in \mathbb{Z}$, let $A_{p q} \in \mathbb{C}(X, Y)$ be the Laurent polynomial defined by

$$
A_{p q}(X, Y)=X^{p+q+2} Y^{q+1}+X^{-q-1} Y^{p+1}+X^{-p-1} Y^{-p-q-2}-(X \rightleftharpoons Y)
$$

$A_{p q}$ is antisymmetric under the exchange of $X$ and $Y$ and satisfies

$$
A_{-p,-q}=-A_{q p}=\overline{A_{p q}}
$$

The characters of $G$ are the polynomial functions

$$
\chi_{\lambda}=\chi_{p q}=\frac{A_{p q}\left(L_{1}, L_{3}\right)}{A_{00}\left(L_{1}, L_{3}\right)}
$$

One easily checks the following identities

$$
\begin{aligned}
\chi_{00} & =1 \\
\chi_{q p} & =\overline{\chi_{p q}} \\
\chi_{p,-1} & =\chi_{-1, q}=0 \\
\chi_{10}(M) & =x+y+(x y)^{-1}=\operatorname{tr}(M) \\
\operatorname{Re}(\operatorname{tr}(M)) & =\frac{\chi_{01}+\chi_{10}}{2}(M)
\end{aligned}
$$

Since $G$ is compact, and $v$ is a continuous function on $G$ which is invariant by conjugation, we can apply the Peter-Weyl theorem to approximate $v$ uniformly on $G$ by linear combinations of characters of $G$. The Fourier transform of $v$ is the function on $\widehat{G}$ defined by

$$
\begin{aligned}
\widehat{v}: \widehat{G} & \longrightarrow \underset{\lambda \in \widehat{G}}{\bigoplus} \operatorname{End}\left(V_{\lambda}\right) \\
\lambda & \longmapsto \widehat{v}_{\lambda}=\int_{G} v \pi_{\lambda}
\end{aligned}
$$

where $R_{\lambda}=\left(V_{\lambda}, \pi_{\lambda}\right)$ is a unitary representation of $G$ for each class $\lambda \in \widehat{G}$ and $d_{\lambda}=\operatorname{dim}\left(V_{\lambda}\right)$. Since $v$ is a class function, Schur's lemma implies that $\widehat{v}_{p q}$ is a dilatation on $V_{\lambda}$. Its coefficient will still be written $\widehat{v}_{p q}$. Moreover, Weyl's integration formula expresses $\widehat{v}_{p q}$ as an integral over $T$ :

$$
\begin{aligned}
\widehat{v}_{p q} & =\int_{G} \chi_{p q} v \\
& =\frac{1}{6} \int_{T}\left|A_{00}\right|^{2} \chi_{p q} \exp \left(\alpha\left(\chi_{10}-3\right)\right) \\
& =\frac{e^{-3 \alpha}}{6} \int_{T} \overline{A_{00}} A_{p q} \exp \left(\alpha \chi_{10}\right) \\
& =\frac{e^{-3 \alpha}}{6} \sum_{r, s \in \mathbb{Z}} C_{\lambda s r} f_{r s}(\alpha)
\end{aligned}
$$


The matrix $C_{\lambda}$ has a finite number of non-zero entries and is obtained by expanding $\overline{A_{00}} A_{p q}$ :

$$
\begin{aligned}
C_{\lambda s r}= & \int_{T} \overline{A_{00}} A_{p q} X^{-r} Y^{-s} \\
\sum_{r, s \in \mathbb{Z}} C_{s r}^{\lambda} X^{r} Y^{s}= & \left(X^{-2} Y^{-1}+X Y^{-1}+X Y^{2}-Y^{-2} X^{-1}-Y X^{-1}-Y X^{2}\right) \\
& \times\left(X^{p+q+2} Y^{q+1}+X^{-q-1} Y^{p+1}+X^{-p-1} Y^{-p-q-2}\right. \\
& \left.\quad-Y^{p+q+2} X^{q+1}+Y^{-q-1} X^{p+1}+Y^{-p-1} X^{-p-q-2}\right)
\end{aligned}
$$

The functions $f_{r s}(\alpha)$ are defined, for all $r, s \in \mathbb{Z}$, by

$$
\begin{aligned}
f_{r s}(\alpha) & =\int_{T} L_{1}^{r} L_{3}^{s} \exp \left(\alpha \chi_{10}\right) \\
& =\int_{0}^{2 \pi} \frac{d \theta_{1}}{2 \pi} \int_{0}^{2 \pi} \frac{d \theta_{2}}{2 \pi} e^{i\left(r \theta_{1}-s \theta_{2}\right)} \exp \left(\alpha\left(e^{i \theta_{1}}+e^{i\left(\theta_{2}-\theta_{1}\right)}+e^{-i \theta_{2}}\right)\right) \\
& =\sum_{n=0}^{\infty} \frac{\alpha^{n}}{n !} \sum_{k=0}^{n}\left(\begin{array}{l}
n \\
k
\end{array}\right) \sum_{\ell=0}^{k}\left(\begin{array}{l}
k \\
\ell
\end{array}\right) \int e^{i \theta_{1}(k+\ell-n+r)} \int e^{i \theta_{2}(2 k-\ell-n-s)} \\
& =\sum_{n=0}^{\infty} \sum_{k=0}^{n} \sum_{\ell=0}^{k} \frac{\alpha^{n}}{(n-k) ! \ell !(k-\ell) !} \delta_{r, n-k-\ell} \delta_{s, 2 k-\ell-n}
\end{aligned}
$$

In the last sum, the non-zero terms are those which satisfy

$$
0 \leq \ell=\frac{n-2 r-s}{3} \leq k=\frac{2 n-r+s}{3} \leq n
$$

Our sums being indexed by the integers for which all the factorials are meaningful, we have

$$
\begin{aligned}
f_{r s}(\alpha) & =\sum_{n \in 3 \mathbb{N}-r+s} \frac{\alpha^{n}}{\left(\frac{n+r-s}{3}\right) !\left(\frac{n+r-s}{3}-r\right) !\left(\frac{n+r-s}{3}+s\right) !} \\
& =\sum_{m \geq \max (0, r,-s)} \frac{\alpha^{3 m-r+s}}{m !(m-r) !(m+s) !}
\end{aligned}
$$

We recognize generalized hypergeometric series [2], which are defined, for $\mu, \nu \in \mathbb{N}$ and $0 \leq \mu \leq \nu$, by

$$
\begin{aligned}
&{ }_{\mu} F_{\nu}: \mathbb{C}^{\mu} \times \mathbb{C}^{\nu} \times \mathbb{C} \longrightarrow \mathbb{C} \\
&(a, b, z) \longmapsto{ }_{\mu} F_{\nu}(a, b, z):=\sum_{n=0}^{\infty}\left(\frac{\left(a_{1}\right)_{n}\left(a_{2}\right)_{n} \cdots\left(a_{\mu}\right)_{n}}{\left(b_{1}\right)_{n}\left(b_{2}\right)_{n} \cdots\left(b_{\nu}\right)_{n}}\right) \frac{z^{n}}{n !} \\
&\left(a_{i}\right)_{n}=a_{i}\left(a_{i}+1\right) \cdots\left(a_{i}+n\right)
\end{aligned}
$$

We thus have

$$
\begin{aligned}
f_{r s}(\alpha) & =\left(\frac{\alpha^{s-r}}{\Gamma(-r) \Gamma(s)}\right){ }_{0} F_{2}\left(\varnothing,(-r, s), \alpha^{3}\right) \quad \text { if } \quad r<0 \quad \text { and } \quad s>0 \\
& =\left(\frac{\alpha^{|r+s|+M_{r s}}}{\Gamma(|r+s|) \Gamma\left(M_{r s}\right)}\right){ }_{0} F_{2}\left(\varnothing,\left(|r+s|, M_{r s}\right), \alpha^{3}\right) \quad \text { if } \quad r>0 \quad \text { or } \quad s<0
\end{aligned}
$$

where $\varnothing$ denotes the empty family and $M_{r s}=\max (r,-s)$. When the argument of one of the $\Gamma$ functions vanishes, i.e. when $r=0$ or $s=0$ or $s=-r<0$, the $\Gamma$ factor is replaced by 1 and the corresponding factorial coefficient in the series starts at the value 1 . Condensing our formulas, we obtain : 
(6)

$$
\widehat{v}_{\lambda}=\frac{e^{-3 \alpha}}{6} \operatorname{tr}\left(C_{\lambda} f\right)
$$

\section{REFERENCES}

[1] J. Frank Adams : Lectures on Lie Groups ;

The University of Chicago Press, 1969.

[2] G. E. Andrews, R. Askey \& R. Roy : Special functions ; Cambridge University Press, 2001.

[3] R. Attal : Combinatorics of non-Abelian gerbes with connection and curvature ; math-ph/0203056 ; to appear in Annales de la Fondation Louis de Broglie.

[5] L. Breen and W. Messing : Differential Geometry of Gerbes ; arXiv: math. AG/0106083.

[6] J.-M. Drouffe and J.-B. Zuber : Strong coupling and mean-field methods in lattice gauge theories ; Phys. Rep. 102, 1983, pp. 1-119.

[7] J. Fuchs and C. Schweigert : Symmetries, Lie Algebras and Representations ; Cambridge University Press, 1997

[8] R. Gambini and J. Pullin : Loops, Knots, Gauge Theories and Quantum Gravity ; Cambridge University Press, 1996

[9] H. A. Kramers and G. H. Wannier : Statistics of the two-dimensional ferromagnet ; Phys. Rev. 60, 1941, pp. 252-262.

[10] S. Mac Lane: Categories for the Working Mathematician; Springer-Verlag, 1997.

[11] I. Montvay and G. Münster : Quantum fields on a lattice ; Cambridge University Press, 1994.

[12] R. Oeckl : Generalized Lattice Gauge Theory, Spin Foams and State Sum Invariants hep-th/0110259.

[13] R. Oeckl and H. Pfeiffer : The dual of pure non-Abelian lattice gauge theory as a spin-foam model ; Nucl. Phys. B 598, No. 1-2, 2001, pp. 400-426; hep-th/0008095.

[14] K. Osterwalder and E. Seiler : Gauge Field Theories on a Lattice ; Ann. Phys. 110, 1977, pp. 440-471;

[15] A. M. Polyakov : Gauge fields and strings ; Harwood Academic Publishers, 1987.

[16] J.- P. Serre : Représentations linéaires des groupes finis ; Hermann, $3^{\text {e }}$ édition, 1978.

[17] K. Wilson : Confinement of quarks ; Phys. Rev D 10, 1974, pp. 2445-2459.

[18] L. G. Yaffe : Confinement in $S U(N)$ lattice gauge theories ; Phys. Rev D 21, 1980, pp. 1574-1590.

[19] R. J. Zimmer : Essential Results of Functional Analysis ; The University of Chicago Press, 1990.

E-mail address: attal@lpthe.jussieu.fr

Laboratoire de Physique Théorique et Modélisation Université de Cergy-Pontoise

5, Mail Gay-Lussac

F-95031 Neuville-sur-Oise (FRANCE) 